\section{THE RADICAL CURE OF FEMORAL AND INGUINAL HERNIA.}

BY C. B. LOCKWOOD, F.R.C.S. ENG., ASTSTANT SURGEON IN ST. BARTHOLOMEW'S HOSPITAL; SURGEON TO THE GREAT NORTHERN CENTRAL HOSPITAL.

1. Genercul considerations.-.The following notes of the radical cure of inguinal and femoral hernia are founded upon upwards of 57 operations. Of these, 45 were performed on non-strangulated herniæ, and 12 after kelotomy. I have also availed myself of the opportunities $I$ have had of watching and assisting at the practice of my colleagues at St. Bartholomew's and the Great Northern Hospitals. It is now quite unnecessary to begin with a defence of the operation of radical cure of hernia. It is at present fully recognised that it is a great boon and that in properly selected cases it gives results equal to those of other operations in surgery. The task before surgeons now is : firstly, the selection of the right class of cases; secondly, the selection of the operation best suited to the case; and, thirdly, the prevention of suppuration. The following cases are published as an aid to the solution of these problems. I propose to mention operations on femoral herniæ first, and afterwards those upon inguinal herniæ in males and in females. Before undertaking or recommending the operation of radical cure it is important to realise what it can and what it cannot do. In the first place, no operation on the abdominal wall can deprive a human being of his predisposition to hernia. I have never examined an abdomen in which an extrusion of the intestines could not occur. The mesentery is always long enough to permit them to enter one or other of the hernial apertures. ${ }^{1}$ All that one can hope to achieve by an operation is to make the abdominal wall as strong as it is in ordinary individuals; but inasmuch as all are liable to hernia it would be rash to promise patients that they would never again have one, or that relapse could never occur. Thus it happens that when this has been explained the patients may elect to try the alternative treatment by trusses. This is not free from disadvantages. It is expensive and troublesome, and trusses not infrecuuently fail at critical moments. The apparent cure which is sometimes effected is not to be relied upon. Amongst my notes of strangulated herniæ many such as the following occur : "Had a hernia in infancy which got well, but came down suddenly at twenty-five and was acutely strangulated"; or, "wore a truss for nine months when an infant, but hernia relapsed again at the ages of eleven, thirteen, and sixteen "; or, again, "ruptured in infancy and wore a truss until five years old ; at the age of twenty-five was lifting, when rupture suddenly returned and was acutely strangulated," and so forth. Of course it may be argued that these were new hernix, but it is more reasonable to suppose that the processus vaginalis had never properly closed. The chance of a cure by trusses becomes decidedly less as age advances, and is improbable in acquired hernia; further, there can be no doubt that after a truss has been worn the local conditions are less farourable for a radical cure. There is much more matting of the sac with the cord or surrounding tissues, and after a time a number of bursal spaces develop, which may be very embarrassing and to some extent may prevent an efficient operation.

As the measures required to prevent suppuration are the same for each class of case they will be mentioned first. It is nsually believed that radical cures are particularly prone to suppuration; however, I feel sure that by careful organisation it may uitimately be banished even from hospital practice. A glance at the details of the cases will show how infrequent it has been, and when it has occurred there has been no difficulty in tracing it to its source. For its avoidance the most minute care is necessary on the part of the surgeon and of all who have to do with the operation. Heat and chemicals are relied upon for disinfection and asepsis. As regards the chemicals it may be noticed that several are in use, but at present I think that carbolic acid for the instruments and biniodide or perchloride of mercury for the rest are the most suitable. Whatever chemical is employed, it ought to be used with a clear knowledge of its mode of action and of the strength required; in other words, the operator ought to know

1 Vide Hunterian Lectures on the Pathology \&c. of Hernia, by accurately what his chemicals can and what they cannot do. The following is now the routine of an ordinary operation. On the preceding evening the patient has a bot bath; the part is carefully shaved and washed with soft soap and water, and its sebaceous matter is removed by ether; next it is disinfected with perchloride or biniodide of mercury in alcohol (1 in 1000 for the former and 1 in 2000 for the latter), and covered with a carbolic gauze dressing, the layers next to the skin baving been soaked in glycerine of perchloride ( 1 in 2000) or biniodide of mercury and glycerine ( 1 in 4000), or in 1 part of carbolic to 40 parts of glycerine. A very large area of the abdomen is treated in this way. The hands of the operator and of his assistants are prepared by cutting the nails, scrubbing with soap and bot water, and soaking for a minute in a solution of perchloride of mercury in spirit ( 1 in 500). The instruments and silk are boiled in water for not less than fifteen minutes and are placed in carbolic lotion (1 in 40) in a sterilised dish or basin, from which they are taken by the operator, used, and replaced without contamination. Sterilised silk has always been used for the deep sutures and ligatures, but silkworm gut has certain advantages for the skin sutures The field of operation is surrounded with clean mackintoshes covered with towels which have been sterilised by steaming for half an hour, with subsequent soaking in carbolic lotion ( 1 in 40). The penis is wrapped in a layer of gauze soaked in the same solution. The nurse hands the sponges to the operator or his assistant in a bowl of solntion of biniodide of mercury. This drug is now used because it seems to have the advantage of not coagulating albumen, and therefore the sponges can be washed in it and kept sterile. Before being used the sponges are prepared by removing the sand by beating and shaking; any shell or coral is removed with dilute hydrochloric acid; and bleaching and disinfecting are done with sulphurous acid solution ( 1 in 4$)$. When not in use they are kept in fresh carbolic lotion ( 1 in 20) in a well-stoppered glass jar, and the lotion is changed onee a fortnight. The only special instruments which are required for the radical cure of either inguinal or femoral hernix are two of Professor Macewen's blunt curved needles. The best patterns are those in which the handle is made with a loop like that of an ordinary wire buttonhook. The operation is conducted as if it were a bacteriological experiment. As little as possible is given to the assistant 10 do, and all hæmorrhage is staunched at once. At the exid the wound is thoroughly disinfected with lotion and closea. The skin is dusted with iodoform, and a layer of carboluc gauze, which has been well disinfected by soaking for some hours in a weak disinfectant ( 1 in 40 carbolic lotior. 1 in 2000 perchloride of mercury lotion, or 1 in 4000 biniodide of mercury lotion), is applied; this is covered with alembroth wool and the usual eight-fold Listerian outside dressing. This dressing is shaped so as to fit rouud the thigh with a strap and buckle, and round the pelves with a similar contrivance. It is secured with a carefulty applied spica bandage, which also exerts moderate pressure upon the wound. Unless these precautions are taken groun dressings are apt to become displaced, with subsequent suppuration. The after-treatment of the operation of radionl cure is simple. I always stipulate before undertaking a case that the patient shall remain three weeks in bed, three weeks on a couch, and have six weeks' gentle exercise. After three months have been passed in this way the patient is allowed to do as he pleases. If the aponeurosis of the external oblique muscle has been divided it is wise to prolong the rest in bed by another week. I feel sure that relapses are due to a disregard of these precautions. For twelve hours after the operation the patient merely requires a little water, which may be warm, tepid, or aerated, and a little cold meat essence. During the next twenty-four hours a little beel-tea bread-and-milk, milk and soda-water, or tea and bread-andbutter are allowed. Afterwards fish and custard pudding and by the fourth day an ordinary diet may be given. As a rule there is no complaint of any pain in the wonnd, but usually the upper part of the abdomen is said to feel stiff and to ache as if it had been beaten. Should there be any real pain after the operation a dose of morphia may be given, but this is not often required. As nearly all the wounds-more than 85 per cent. of the non-strangulated cases - heal by first intention, there is hardly any disturbance of the general health, and reading and like pastimes may be indulged in.

2. Femoral hernia. - The operation which I have performex upon femoral hernix is the same as that which most surgeous practise, but with an additional device for closing the femoral 
canal, which, as far as I am aware, is not generally used, but which is very efficacious. Briefly, the procedure is as follows: The sac is exposed by a vertical skin incision kept, for the avoiannce of infeotion, well away from the organs of generation, and extending an inch above Poupart's ligament and two inches down the thigh. The first precaution renders asepsis easier, and the second facilitates the introduction of the deep sutures. In very fat persons a swall sac may be difficult to find, but the spine of the pubes is a sure gaide to it. The sac is opened and its contents are dealt with in the manner described below. Next it is freed from its surroundings, especially from the femoral canal, and its neck is transfixed and secured with a stout silk ligatule expeditiously tied in a Staffordshire knot. The ends of the knot are left long and are used to draw the neck of the sac into the abdomen and to fix it there. This is done by threading each Jigature in turn upon a Macewen's needle, which is passed up the femoral canal, along the subperitoneal tissue, and thrust through the abdominal wall about the ruiddle of Hesselbach's triangle and just above the inguinal canal and its contents. As these sutures are passed the skin and subcutaneous fat are pulled up out of the way of the needle, which emerges through the aponeurosis of the external oblique muscle, where it is unthreaded and the silk is tied in a firm knot upon the surface of the aponeurosis. When the skin is released the knot is buried beneath it. In passing these needles up the femoral canal the common

Frg. 1.

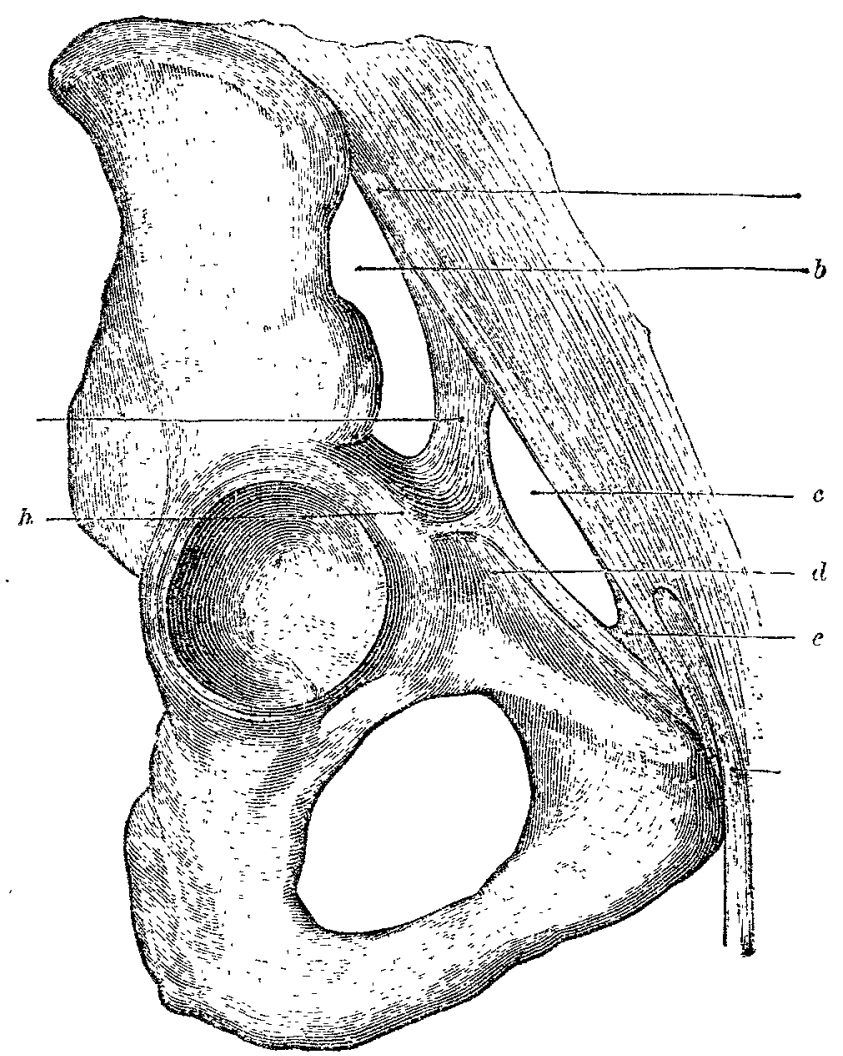

$a a^{2}$ Poupart's ligament. $b$, Lacuna muscularis. c, Lacuna Pascuiaris. $d$, Cooper's ligament. $e$, Gimbernat's ligament Spermatic cord. $g$, Ilio-pectineal ligament. $h$, Iliopectineal eminence. Note. $-T$

femoral vein is protected with the finger and the point of the needle is turned inwards. After this stage the neck of the sac ought to be inside the abdomen, having been pulled up by the sutures, and the femoral canal ought to be quite clear and erapty. To close the femoral canal I have been accustomed of late to fasten together Hey's and Cooper's ligaments. By the fermer is meant the continuation of the falciform process of Burns, which runs inwards towards the spine of the pubes and forms the roof of the femoral canal; the latter is as strong fibrous band ${ }^{2}$ which, as is shown in the accompseying fgure from Professor Macalister's book on Anatomy, runs along the pectineal ridge from the spine of the pabes to the ilio-pectineal eminence and gives attachment to the pectineus muscle and to the fascia which covers it. Cooper's ligament is of consider-

2 See A Test book of Truman Anatomy, by Prof. Alexander Macalister 1889, Fig. 261, p. 170. Sce ilso Sappey : Traité d'Anatomio Descriptive, $18 \% 6$, p. 405 et sea. able strength and gives a very strong hold to the sutures placed beneath it. These are passed as follows. With a Macewen's needle one end of a strong silk ligature is thrust from within outwards through Hey's ligament at its junction with Poupart's ligament. To accomplish this the needle is passed up the femoral canal. The other end of the same ligature is next carried with the same needle from within outwards between Cooper's ligament and the pectineal crest of the pubes. In performing this manceuvre the common femoral vein is protected with the finger, and to pick up the ligament the point of the needle is scraped over the edge of the ridge of bone. Sometimes one suture is sufficient to close the $f \in m$ moral canal, but as a rule two or even three are required. As I have already said, the ligatures have an exceedingly strong hold, and when they have been tied the femoral canal is quite occluded. Whether there is any danger of compressing the femoral vein I hardly know, but the possibility must be taken into account in passing the sutures. The sutures must be firmly tied, and it is better to diminish their number rather than to tie them loosely. This operation is not difficult to perform, but I have seen, though not experienced, difficulty in finding the sacs of femoral hernix on account of their smallness or because of the fatness of the patient. Sometimes, owing to the sac having become converted into a multiplicity of cysts by inflammatory changes, it is hard to find the opening into the abdomen. In one of my cases two blind cysts containing yellow serum were explored before the third, which opened into the peritoneal cavity, was found. Also the sac may be hidden by a mass of fat surrounding its exterior. In one case a tumour which felt like an irreducible epiplocele was found to consist of a mass of subperitoneal fat with a small sac in its centre. In another case the hinder wall of the sac was very thick and fleshy, simulating the presence of some abdominal viscus; this condition was met with in a slighter degree in anotber case. Most of the femoral hernix contained adherent omentum, or omentum and fluid, or fluid alone. As a rule the adhesions were easily broken down with the finger, but sometimes those near the neck of the sac were troublesome to undo. In a case of double femoral epiplocele sent to me by Mr. Fraser Stokes I found a large piece of omentum in the right sac. This was transfixed and ligatured with a Staffordshire knot; but the pedicle was so large that great force was required to thrust it into the abdomen. Since this, whenever there has been much omentum it has been secured with an interlocking chain ligature, such as is described in works on surgery. ${ }^{3}$ It requires practice to introduce this ligature neatly and quickly, but it is secure and does not make a large pedicle. A bulky omental pedicle has other disadrantages. In the case I have just mentioned and in another one it seemed for a time to cause some abdominal discomfort, and it was thought that a tender spot marked its site. I have never seen suppuration around it, such as is described by LucasChampionnière in some of his cases. 4 The omentum is a structure to be treated with great care. In my Hunterian Lectures I mentioned fatalities due to ligatures slipping. Since then I have seen a case in which the operator unwittingly tore through some of the omental vessels near the stomach whilst pulling the omentum down; in consequence the peritoneal cavity became full of blood, and the patient ultimately died. Twelve operations have been performed for the cure of nonstrangulated femoral hernize, two of the patients being males and the rest women. The only fatality occurred in this class and was due to acute bronchitis after the ether. The patient succumbed on the sixth day. The wound was almost healed by first intention, and it was clear that the operation had firmly closed the femoral canal and ring without leaving a peritoneal depression. It was impossible to force the finger down the femoral canal into the thigh. Although I blame the ether for this most unfortunate calamity, I have to add that severe bronchitis occurred in another case after the administration of chloroform in an operation for the radical cure of both an inguinal and of a femoral hernia in the same person and at the same time. The operation was therefore a prolonged one, and the chloroform had to be given for over an hour. In the case of ether bronchitis the operation was brief, but a history of a winter cough was afterwards obtained. of the eleven cases which remain ten healed by first intention. One dressing was enough for each, with the exception of an early one, which was drained and in consequence was

For instance, Greig Smith: Abdominal Surgery, p. 139, Figs. 27, 28 , and 99,1888

4 Cure Radicale des Hernies, p. 448 et seq. Paris, 1892. 
dressed a second time for the removal of the tube. The operation was performed on Jan. 10th, 1888, since which date no case of femoral hernia has required to be drained. In another case a second dressing was required because the patient, who was neurotic, conplained of pain, so the was found and the wound healed by first intention under the second dressing. The only case which did not heal by first intention had very slight superficial suppuration. No silk came out, and the highest temperature was $99.2^{\circ} \mathrm{F}$. The wound was dressed on the tenth day and after having been dusted with iodoform and dressed with alembroth wool gave no further trouble. The operation was carried out on Web. 2 nd, 1891 , and she left the hospital on March 12th with the wound healed and without a truss. Silk sutures and ligatures were used for all these cases, and in none of them did any of them come out.

Two men were operated upon for the radical cure of femoral hernia. The operation in men is rather more difficult than in women, because the femoral canal is not so large and the structures are rigid, so that it is more diffenlt to manipulate the needles in putting in the deep sutures; also the presence of the spermatic cord just above the femoral canal is an embarrassment and has to be shunned. Both of these cases healed by first intention, and the abdominal wall now seems to be very strong and without impulse. One of these men had an inguinal hernia operated upon at the same time, and both wounds healed by first intention. He was obviously a bad subject for a permanent cure, but he was very anxious to have it done so as to enable him to secure the advantages of a club. The operations in this class are not very numerous because as a rule those who have small and easily reducible femoral herniæ prefer to be treated with trusses. This will usually suffice, as they are generally women who are little exposed to strains, violence, or heavy lifting ; also, an operation upon reducible femoral hernia is not to be strongly urged, because in a certain number the lesion is not, like so many inguinal ruptrures. due to a local congenital defect of the abdominal wall, and still therefore less capable of being cured by local measures. The rupture is in some cases of femoral hernia a part of a general defect of the tissues of the peritoneum and of the suspension of the viscera. Nevertheless, there are many which seem to be due to local remediable causes, and in these a cure can usuaily be obtained. There is a large class of patients with irreducible femoral epiploceles and with socalled femoral hydroceles, to whom the operation of radical cure is the greatest boon, and upon whom it can be strongly urged. Such patients are most grateful to be relieved of their pain and discomfort, and to be freed from those illadapted cupped trusses, that are sometimes ordered; indeed, one of my cases was wearing one of these things when strangulation occurred. Also there are many young women to whom any kind of truss is irksome, and who when they are offered the alternative of an operation gladly embrace it in the hope of obtaining a permanent result. After kelotomy a radical cure prevents immediate recurrence, subsequent relapse, protrusion of the sac, suppuration in the sac, sloughing of the sac, and peritonitis spreading from the sac. ${ }^{5}$ Therefore a proper radical cure has been effected five times after kelotomy for strangrulated femoral hernia. Four of these cases bealed by first intention under a single dressing. One patient died, but there was no clear eridence to show that the untoward result was caused by the radical cure. The details of this case are instructive and are briefly as follows. A woman sixty-seven years old had had a right femoral hernia strangulated for fifty-six hours. At the operation the gut was in good condition and was replaced, a radical cure being afterwards eflected. For two days there was complete relief with the passage of flatus and the cessation of vomiting. However, the symptoms of strangulation then recurred and were attributed to peritonitis, as a tender and indurated swelling could be felt in the iliac fossa. The patient died, and a knuckle of gangrenous intestine was found prolapsed through a rent in the peritoneum not far from the original neck of the sac. $X$ greatly regret that I did not perform an exploratory laparotomy in this case as soon as these symptoms of strangulation recurred. I have not included in this class some cases in which it was deemed expedient merely to pass a ligature round the neck of the sac; $\mathrm{as}_{\text {, for instance, was }}$ done in the case of a bed-ridden old woman operated on for

5 On the Importance of performing the Radical Cure after operations for Strangulated Ifernia, by C. B. Loekwood. Brit. Med. Jour., July 8 th, 1891.
Dr. Wight, or in that of a very old man operated on for Mr. Frank Godfrey.

3. Inguinal hernia.-As far as possible I have confined the operation for the radical cure of inguinal hernia to the congenital hernix of young adults, the age of the patients averaging. eighteen years and eight months. As I bave endeavoured to show elsewhere, ${ }^{6}$ these may, on anatomical and pathological grounds, be expected to give the best. and most permanent results. Mr. A. Quarry Silcock, who worked upon theso lines, brought to the Harveian Society the best series of cured cases which has yet been shown in London. ${ }^{7}$ I have only twice to my own knowledge operated on acquired inguinal hernix-once to enable the patient to wear a truss and once to enable him to benefit by a club to which he belonged. In congenital hernia there is no morbid delect of the peritonoum or mesentery, or suspension of the abdominal viscera, but only a local developmental defect of the abdominal wall, capable of being completely remedied by operation. A most favourable result may confidently be anticipated when there is a long, narrow congenital sac occupied by a thin strip of omentum. On the other hand, the aged and middle-aged with accuired hernix owe their ruptures to causes which cannot be removed by an operation upon the abdominal wall. Their tissues are defective and deteriorated, and the ruptures are often associated with simple or complicated prolapse of the mesentery. I have occasionally operated on this class, but only to enable the patient to wear a truss. In one man two very large hernix were operated on at an interval of a fortnight. The right sac contained the cæcum, vermiform appendix, and ileum; the left was empty. Both wounds healod under a single dressing, and the patient was able to wear a double scrotal. truss and to earn his living again. I would once more urge that the choice of cases for radical cure in non-strangulated hernia should be made on anatomical and pathological grounds and with clear and precise knowledge of the disease; too many unsuitable cases have been operated on. Children, too, are not very suitable for operation. Their tissues are delicate and hard to manipulate, their wonnds are difficult to. keep aseptic, and they are not infrequently cured by trusses. However, I do not hesitate to operate uron this class if their ruptures are small or if they cannot wear trusses. Inguinal hernix in little girls seem to be particularly favourable and give excellent results.

A great number of the cases of inguinal hernia which I bave operated upon were in reality operations of necessity and not of expediency. Some of the patients could not wear a truss because of adherent omentum, some on account of irreducibility, others because of malposition of the testis; several, in spite of trusses, had bad strangulation and did not wish to incur the risk of it again; some were operated upon to enable them to enter or to remain in the army; and, lastly, some few because, having seen the benefit in others, they voluntarily preferred the risk and inconvenience of an operation to the troubles of a truss. Thlis as an increasing class. The points to be aimed at for the radical cure of inguinal hernia are now so well known as hardly to require to be mentioned. The first is the obliteration of the sac without leaving a depression at its opening into the peritoneal cavity, and the second is the restoration of the inguinal canal Inguinal berniæ differ so much from one snother that I am now accustomed to graduate the operation which is required to fulfil these conditions according to twhat is met with at the time of operation. The cases in which the inguinal canal was freely opened were those in which the hernia was incomplete or in which there was a retained testis, or in which a large and negleoted rupture had so stretched and obliterated the canal that an efficient operation could not be carried out through the unenlarged extersal abdominal ring. In the last class the inguinal canal hat yaite lost its valvalar arrangement, its posterior wall having been dragged inwards towards the middle line by the trastion of the hernia until the finger could be thrust straight into the abdomen. The operations on inguinal hernix may we divided into two classes : (1) those in which the inguinal canal was not opened, the aponeurosis of the external oblique ravasle having been preserved intact; and (2) those in whic's the inguinal canal was freely opened by division of the aponeurosis of the external oblique muscle. Those who are familiax with the somewhat extensive literature of this subject will see that in carrying out the procedures I have freely availed myself of the

6 I Iunterian Lectures on Hernia, 1989, p. 104 7 Clinical Jour nal, vol. ii. 1,42 . 
operations devised by Mitchell Banks, Macewen, Ball, Barker, Kocher, Bassini, and Halstead. The first operation, which I shall in future call the "ordinary" one, was done in cases in which the hernial sac was long and narrow, and either empty or occupied by a slender piece of omentum, often slightly adherent. In this class, which is the most favourable one, after the sac had been opened and the omentum cut away, the finger had to be thrust a long way up the canal before it arrived at the mouth of the sac, which latter had not been dragged inwards, but lay opposite the centre of Poupart's ligament; also, when the finger was thrust against the back of the inguinal canal, it was found to be intact and in its proper position. The details of the ordinary operation are briefly as follows. The whole length of the front of the inguinal canal is exposed by a straight incision three-quarters of an inch above and parallel to the inner half of Poupart's ligament. The greatest sare is taken to shun the scrotum. As the skin of the groin is loose it is quite easy by displacing it to get at anything through the incision. The sac is opened as it emerges from the external abdominal ring and explored with the finger, and its contents are dealt with. If the omentum is present it is treated with great consideration and the same as in femoral hernia. Next, if the sac is a congenital one or very long, it is cut across opposite the external ring and its lower end is dropped into the scrotum, never to be seen or heard of again; int if the sac is short it is unnecessary to cut it across. In in $n$ case its upper end is separated from the spermatic cord 2.8 far as the peritoneum. As a rule it is best to pull the constiments of the cord away from the sac with dissecting forceps. whe separation is easier as the internal ring is approached and it must be thoroughly done; later it will be seen that the neglect of this precaution has led to a relapse. The upper end of the sac is now pulled down, usually twisted upon itself, transfixed with a blunt needle armed with stout twisted silk, and is tied high up at the internal abdominal ring with a Staffordshire knot. Any redundancy of the stump is cut away, and the ends of the silk, which have been left long, are used to fix the stump of the sac beneath the fascia transversalis, and, more particularly, beneath those muscular fibres of the internal oblique muscle which arise from Poupart's ligament, and which in future, for the sake of brevity, I shall call the "arciform fibres." The accompanying figure from

Fig. 2.

(From Cunningham.)

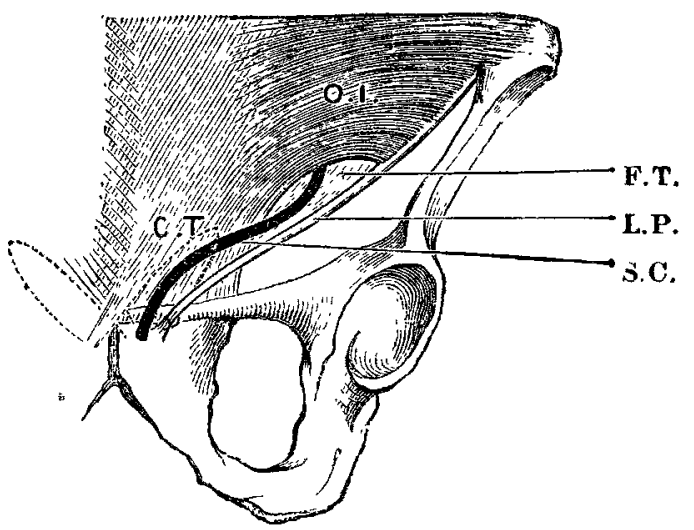

a.r., Internal oblique muscle. C $T$., Conjoined tendon. F. T., Fascia transversalis. L P , Poupart's Jigament, stump of the sac.

Professor Cunningbam's ${ }^{8}$ admirable work shows theso fibres, and the position of the stump would be just below the letter $O$ I., acd, of course, hidden from sight by the arciform fibres. A finger is used to make a bed for the stump of the sac in that position, and it is fixed with the ends of the silk ligature wbich encircles it. This is done by carrying the ends separately up the inguinal canal with a Macewen's needle which is forced in two places a little way apart through the fiscia transwersalis, arciform fibres, and aponeurosis of the external oblique muscle. The ends of the ligature are unthresded and are tightly tied upon the front aspect of the aponeurosis of the external oblique muscle, the skin wound being palled up to facilitate this. It is obvious that, after the stump of the sac has been fixed in this manner, any impulse imparted to it from within would be expended in driving it against the arciform fibres and the external oblique muscle, both of

B Manual of Practical Anatomy, vol. i., p. 4' 5, Fig. 80. which are structures eminently qualified to prevent extrusion. After the hernia and its sac have been dealt with the inguinal canal is sometimes so perfect that little more remains to be done; but as a rule it is necessary to approximate the conjoined tendon to Poupart's ligament with one or two deep sutures passed as follows. With the blunt curved needle, guided by the finger, a stout silk ligature is passed through the conjoined tendon about halfway up the inguinal canal, care being taken to avoid the peritoneum and deep epigastric artery. The ends of this loop are next carried with the blunt needle throngh the front of the inguinal canal and are tied subcutaneously. The upper end of the loop ought to be passed behind the vas and through the arciform fibres and external oblique muscle; the lower pursues the same course, but being a little lower down the canal it may only pierce the external oblique aponeurosis. When this suture has been properly passed and tied it pulls the conjoined tendon downwards and outwards behind the cord and restores the back of the inguinal canal. Sometimes a second loo $\mathrm{p}$ is required, but if the back of the canal is so defective as to require more than two sutures I prefer to divide the aponeurosis and to perform Bassini's operation. To complete the operation the external ring may require to be closed a little, and finally the skin incision is sutured. It will be observed that little has been said about the internal abdominal ring and about closing it. The introduction of this mythical aperture into surgical writings has been a great bugbear to everyone. It is difficult to manufacture in the dissectingroom and it is not to be seen in the operating theatre. As a phrase, however, it is convenient to mark the point at which the spermatic cord begins to enter the inguinal canal. In cases of small, incomplete inguinal herniæ an operation the same as the ordirary one has been carried out, except that to reach the sac the inner half of the front wall of the inguinal canal has been divided. In operating upon females this is often necessary, or when minor difficulties are met with in separating the sac from the cord or in the introduction of the deep sutures. I have not yet seen any ill-effect ensue from division of the aponeurosis, and it greatly ficilitates the manipulations. It is impossible many months afterwards to tell that it has been divided, the external abdominal ring having in several cases retained the dimensions given to it at the operation. Nevertheless, the aponeurosis ought not to be heedlessly divided. It is obvious that a structure such as it is will take long to repair, and that the patient must be kept in a recumbent position for a longer time than if it had been left intact. The effect of suppuration on its repair need hardly be mentioned; but as that is a strictly preventable occurrence it may be assumed that we shall gradually obtain better success. The ordinary operation was done 17 times upon the inguinal herniæ of males, and of these 14 healed by first intention, 1 had very slight superficial suppuration, and in 2 the suppuration led to the extrusion of some of the deep sutures. The slight case of suppuration hardly delayed the healing of the wound, which only required three dressings and was well on the twelfth day. In this case a misplaced, atrophied testis was also taken away. The slight suppuration was cansed by a small sinus connected with a suppurating gland. Both the other cases of suppuration were thought to be due to ill-prepared silk; they occurred at the same time and under the care of the same house surgeon. None of the cases which healed by first intention required many dressings : 10 bealed under a single dressing; 3 had two dressings because repair had been hindered in 2 of them by in-turned skin, and in 1 ( $\mathrm{a}$ boy) by a drainage-tube which was put in on account of oozing of blood at the time of operation; 1 (a boy) was obstreperous and pulled his dressings of several times, nevertheless his wound healed by first intention. In the 2 cases which extruded some of their deep sutures the suppuration was quite local and subacute and was not noticed until the end of the first week. It was treated with boracic fomentation. They caused no anxiety, and nitrous oxide gas was given about the sixteenth day and the silk was extracted. One of these is known to bave relapsed.

The operation in which the inguinal canal was freely opened can be described very briefly, as it does not differ in principle from the ordinary one. The inguinal canal is exposed by a similar incision, which is carried through its front wall. At this stage care ought to be taken not to injure the arciform fibres of the internal oblique muscle, otherwise a weak abdominal wall may result. After the interior of the canal has been exposed the hernial sac is opened and explored, and its 
contents are dealt with. If the sac is long and goes down into the scrotum it is cut across at the level of the pubic spine, and its lower end is dropped into the scrotum. The upper end is separated as high as the peritoneum, transfixed with a stout silk ligature, and secured with a Staffordshire knot. The ends of this ligature are used to fix the stump of the sac beneath the arciform fibres, where the finger has made a bed for its reception. For this purpose the two ends are carried with the blunt needle through the arciform fibres and the underlying fascia transversalis in the same way as in the ordinary operation; but sometimes I have found it to be rather better not to pass the ligature through the aponeurosis of the external oblique, as is done in the ordinary operation, because this is apt to interfere at a later stage with the approximation of the aponeurosis. After the stamp of the sac has been fixed beneath the internal oblique the spermatic cord is lifted out of the inguinal canal, and the deep sutures, which restore the back of the canal by pulling the conjoined tendon downwards and outwards, are passed in the manner taught us by Bassini. First, the threefold layer, consisting of the internal oblique and transversalis muscles and transversalis fascia, is isolated by pushing the finger between it and the aponeurosis of the external oblique and between it and the peritoneum. Next the spermatic cord is lifted up and the deep internal surface of Poupart's ligament is exposed by the finger or by a blunt instrument. Then, with a Macewen's needle the threefold layer is fastened to the deep surface of Poupart's ligament by from three to six silk sutures, which pierce them both, beginning below near the spine of the pubes, and which are tied inside the inguinal canal beneath the cord. (Fig. 3.) To complete the operation the spermatic cord is returned into the canal, and the aponeurosis of the external oblique is sewn together again and made taut, the wound being closed and dressed. Silk sutures are used throughout, except for the skin, in which silkworm gut is rather better. There can be but little doubt that this operation is thorough, and it has the advantage of being guided by the vision. It may entail, however, some bruising and laceration, and on that account it is more likely to be followed by suppuration unless asepsis is secured. Further, after division of the aponeurosis, it is, I think, necessary to keep the patient in a recumbent position for a longer time.

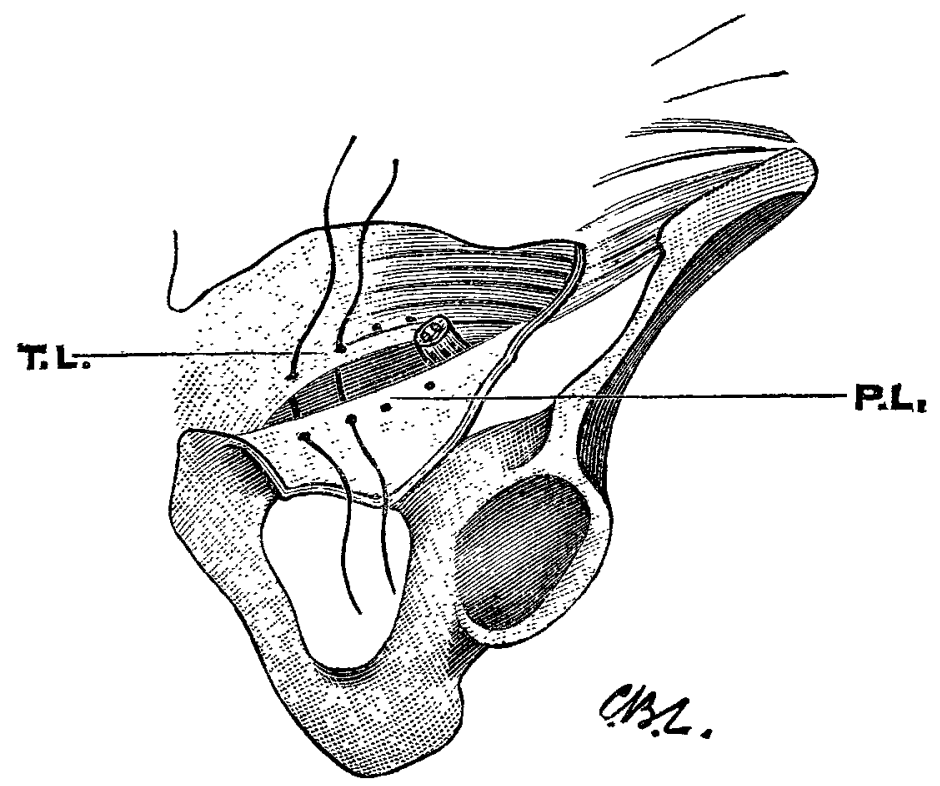

To show the method of inserting the deep sutures after the inguinal canal has been opened. For the sake of clearness the up. P.L., Poupart's ligament. T. L., Triple layer.

The inguinal canal was laid open and Bassini's operation was performed in nine cases of inguinal hernia in males. One of these had late subacute suppuration, with extrusion of the deep sutures, because an inexperienced house surgeon took the dressing off on the fifth day and removed the stitches, as be thought that the wound was healed. Nine months after the operation this patient wrote, in reply to my communication, that he had occasional pains, but was able to work as a gardener and did not require a truss. Another case had subacute suppuration which ended in the extrusion of sutures, and in a third case there was slight stitch-hole suppuration, which was well in three days and was thought to be due to imperfect disinfection of the skin. The remaining six cases healed by first intention under a single dressing.

Malpositions of the testis were met with in five cases of inguinal hernia. In two of these the organ, being very illdeveloped, was excised-a procedure which added to the ease of the operation and made no difference whatever in the recovery. In two cases the testis was brought into the scrotum by inverting it in the manner recently advocated by Mr. Bidwell. In one of these cases the testicle atrophied, but in the other the result was admirable. The gland, although smaller than its fellow, felt quite normal. Recently I assisted my friend and colleague, Mr. Bowlby, to operate on a case in which he was able without trouble to replace an undescended testis in the abdomen and to perform what $I$ have called the "ordinary" operation.

Seven females were operated on for the radical cure of inguinal hernia. In these, owing to the absence of the spermatic cord, the operation was particularly easy, and the result were most favourable. The ruptures were all congenital in their nature, with rather thick-walled sacs and with a small communication with the general peritoneal cavity. Most of the ruptures were incomplete, and therefore the inguinal canal had to be freely opened in five cases and partially in another. After the canal had been opened three or four deep sutures, according to Bassini's method, were enough for its effectual restoration. The ordinary operation was carried out twice.: in one case there was no feature of interest, and the other, as I have just stated, merely required a partial division of the aponeurosis of the external oblique. A hernia of the ovary was met with once, and as both that organ and the Fallopian tube were small and ill-developed they were taken away. Healing by first intention occurred in six instances, but one had to have two dressings, because the skin of the abdomen had been blistered. This was remedied by dusting with iodoform and covering with iodoform gauze. The case was aseptic when tested with cultures. One case suppurated, owing, I believe, to the presence of staphylococcus aureus in the skin. At the operation a bit of skin was put into broth and grew that microbe. The suppuration occurred on the sixth day and caused no anxiety.

Thus of 44 recoveries after the radical cure of non-strangulated hernia 36 healed by first intention; of this number 3 required a single dressing and 5 required two; and 1 , the troublesome schoolboy, required several. Of the remaining 8 cises, 5 had suppuration which ended in the extrusion of some or all of the deep sutures, and 3 had very slight suppuration, which made no appreciable difference in their healing.

Radical cure after operations for the relief of strangulated inguinal hernia does not give such good results, either immediate or remote, as it does in selected cases, operated on at a chosen opportunity. The immediate results are bad on account of the conditions at the time when the operation is done, and the results are likely to be unfavourable because most of the patients have acquired herniæ which, as I have already said, are only a part of their disease. On seven fairly favourable occasions a curative operation was performed after kelotomy for the relief of strangulated inguinal hernia. Although some of these ruptures were of enormous size, ${ }^{9}$ and in one a loop of intestine was resected, they all recovered. I attribute this fortunate result in no small measure to the care with which the strangulated loop was disinfected and te the occlusion of the neck of the sac by the curative opera. tion. The last step is particularly important because sappuration is still common in these cases and occurred in four instances. The causes of the suppuration, which was never of the acute kind and was sometimes very slight, seem to be quite simple, and when known may in future be avoided. For instance, owing to the urgency of the symptoms a proper disinfection of the skin, especially that of the scrotum, could not be carried out with the disinfectants $I$ bave hitherto been accustomed to use. Next, owing to the size of the hernix and the presence of bad adhesions, the scrotum had to be incised; this is always a serious complication. Lastly, owing to the duration of the strangulation, there is no donb that the fluid in the sac was septic. The general condition of these patients, too, was highly unfavourable for healing of their wounds by first intention.

It is a question how many of these cases onght to be included in alist of radical cures. Three of these patients had the ordinary operation performed, but in four the gap left in the abdominal wall after reduction of the gut was so large, and the condition

9 Two contained the sigmoid flexure, each haping one loop free and another adherent in the sac. 
of the patient was so critical, that a thoroughly satisfactory curative procedure could not be undertaken ; therefore the gap was simply closed with a series of five or six stout silk sutures. Each of these included the peritoneum and the whole thickness of the abdominal wall, and the lowest were passed so as to pleat up and obliterate the neck of the sac. As suppuration is apt to occur in these cases it is, perhaps, better not to bury these sutures, but to bring their ends out through the skin in the usual way ; also stout silkworm gut may be advantageously substituted for silk.

The ultimate results of this somewhat crude operation have been better than I expected. One case was very instructive. The patient had a large oblique inguinal hernia upon the right side, which had been strangulated in 1880 and operated upon. No radical cure was effected, and it came down again in spite of a truss. I operated for strangulation on July 1st, 1886, and effected a radical cure. I saw this man on Sept. 2nd, 1893, with a sound abdominal wall, although in the interval he had worked hard and had been spared the inconvenience of a truss. Another man, who had an enormous hernia, said a year after the operation that he was cured and wore no instrument, being able to work as a cabman; but this case ultimately relapsed.

Like other surgeons who practise in London, I have the greatest difficulty in tracing cases; therefore I am only able to illustrate the ultimate results of the operation of radical cure by giving such samples as I have been able to obtain. I claim, however, that they show that the operation of radical cure of inguinal and of femoral hernia is, when efficiently done upon proper cases, as successful as any operation in surgery. A young man aged twenty-two years was operated on on March 16th, 1892, for the cure of a right inguinal hernia. He wished to enter a trade society which would not admit him if he merely wore a truss. On Oct. 20th last his cure was perfect, he could do the hardest work, and had been a member of his trade society for a year. A gentleman who was operated on on April 21st, 1892, was quite well in October last, being able to shoot, ride, row, or do anything he wished. A schoolboy who was operated on on July $17 \mathrm{th}$ 1892, was quite well on Oct. 20th last, and is now able to play cricket and football. I have no cases of Bassini's operation of more than a year's standing, but I have had no relapse in this class, and those who have come up for inspection seem to be perfect. The ultimate results of the operation for the radical cure of femoral hernia have been quite satisfactory, although I can record a relapse in a case in which the neck of the sac was merely tied. One which was performed on a delicate woman fifty years of age, after strangulation, on Jan. 5 th, 1889, showed no tendency to relapse on May 14th, 1890, and she required no instrument until her death, which occurred in the following year. Mr. H. Fraser Stokes reports that a cook aged forty-eight years, who was operated on for right and left femoral epiploceles on Jan. 25th, 1891, was quite well on Oct. 20th, 1893. Also that one of her fellow servants, who bad a small irreducible femoral epiplocele, wished to have the same operation performed. It: would not be easy to find more striking evidence than this of the benefits of the procedure. I have only met with two cases of relapse, although I have taken great pains to keep the cases under observation. In one case the relapse was due to suppuration; it occurred six months after the operation. The patient elected to wear a truss, which he could not do before. The second relapse was due to a faulty operation. I performed the ordinary one on a young man, and six months afterwards his hernia recurred while he was lifting a heavy weight. As the first operation had caused him no pain and very little inconvenience he came back to ask to have it carried out again. As the relapse was very small and high up the inguinal canal the aponeurosis was incised and Bassini's operation was performed. This enabled me to ascertain that the relapse was due to about three-quarters of an inch of sac having been left behind on the first occasion.

Upper Berkeley-street, w.

Iondon Post-Graduate Course.-The vacation courses in Bacteriology, Pathology and Hygiene, have been arranged and will commence on Monday, Dec. 4th, and end on Friday, Dec. 15th. The gratifying success of the September vacation classes shows that there are many practitioners who can only leave their work for ten or eleven days at a time and are anxious to receive further instruction in these important subjects. The class lists are now being made nut, and entries may be made on application to Dr. Fletcher Little, 32, Harley-street, W.
THE

DETACHMENT OF THE EPIPHYSIS FOR THE ANTERIOR INFERIOR SPINOUS PROCESS OF THE ILIUM THROUGH ENFORCED MUSCULAR ACTION.

BY R. HENRY ANGLIN WHITELOCKE, M.B., M.C. EDIN., F.R.C.S. ENG.

SURGICAL literature affords but few examples of the condition which the above heading describes. With the single exception of a case mentioned by Mr. F. Treves, ${ }^{1}$ in which it occurred while the subject was in "the act of running a race," examples are not to hand. For this, among other reasons, it has been considered to be advisable to place on record the clinical details of two cases which have lately occurred in my practice. The hope is also entertained that by so doing cases which may have been observed by others will now be recorded. My belief is that this accident is probably not so rare as at first would seem to be the case. Besides entering inte the clinical details of the cases it is proposed to more fully consider and inquire into the anatomy and etiology of the injury as well as to deal with its treatment. The clinical histories and symptoms of the two cases are so nearly identical that one description might almost suffice for both. One evening early in February, 1891, an undergraduate drove up to my house, and after hobbling into my room gave the following bistory. In starting to run in a race of 100 yards distance, while practising for his college sports, he "seemed to have scarcely travelled ten yards when he had suddenly to stop as he felt something snap, and at the same time his limb became powerless." There was then some pain, but it was " by no means severe," although he " became faint and felt sick." On removing his clothes there was little to be seen excepting that the injured limb assumed the position of semiflexion and was in advance of its fellow, which, being fully extended, was sustaining the whole weight of the patient. When asked to straighten the injured limb by extending the hip- and knee-joints increased pain was felt, and he had extreme difficulty in extending the knee-joint; but what he most complained of was an unpleasant feeling of numbness and tingling perceived all down the extensor surface of the thigh. There was very little discolouration, accompanied by an inconsiderable swelling situated for the most part beneath and to the outer side of the middle of Poupart's ligamentthe region, in short, of the anterior inferior spinous process of the ilium. After carefully comparing the two sides and palpating, a distinct crepitation could be elicited on deep pressure in the region of the swelling: and this could be even more distinctly made out when, with the hands placed over the seat of the injury, the patient made an effort to bring the rectus muscle into action. The morable piece of bone gave one a feeling on palpation not unlike that of a loose cartilage in a joint. The diagnosis was then made, but not before all the usual sites had been carefully examined for a possible her nia the history of something having snapped, the pain, and the feeling of sickness and faintness favouring such an occurrence. A pad with a temporary spica bandage was applied, and the patient was sent back to his college. On visiting him next morning the swelling had somewhat increased, but the discolouration remained about the same. There was pain only on movement, while the tingling sensations persisted unaltered. 'Two days after this another undergraduate from a different college was seen, and he gave an almost similar history. $\mathrm{He}$ had been starting to run "a quarter mile." In the very act of starting - in the first bound, as it were-he "felt something give, and became powerless to proceed." With the experience of the former case it was not difficult to discover what was the matter. In this case a mere scale of bone, as far as could be elicited, came away with the straight head of the rectus, and crepitus was with difficulty made out at first. The tingling sensation and other symptoms described in the former case were also evident.

Thus, the histories and causation of these cases were remarkably alike. Kach patient had started with his left limb somewhat flexed both at the hip- and knee-joints, and in advance of the right, the body being bent forwards and, as it were, 